
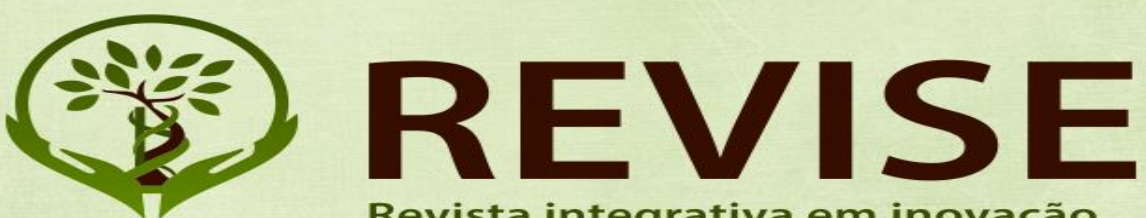

Revista integrativa em inovação tecnológica nas ciências da saúde

ISSN: 2179-6572

\title{
Artigo: WORKSHOP DOS GRUPOS DE PESQUISA DE INOVAÇÃO TECNOLÓGICA NAS CIÊNCIAS DA SAÚDE.
}
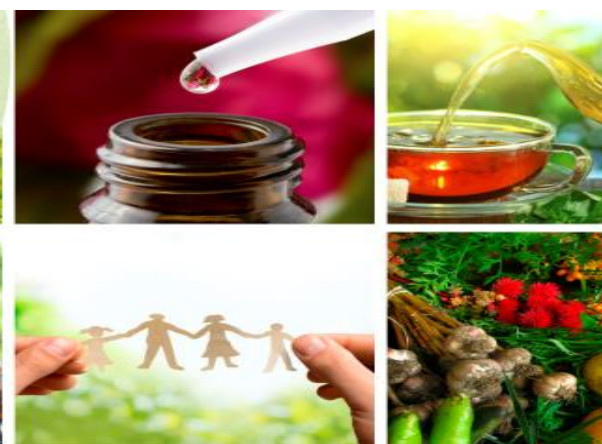

Júlio César dos Santos

$U F R B$

Resumo:

O artigo registra as conferências do evento congresso internacional de inovação tecnológica nas ciências da saúde: a sustentabilidade das práticas integrativas a agroecologia, mais especificamente a conferência WORKSHOP DOS GRUPOS DE PESQUISA DE INOVAÇÃO TECNOLÓGICA NAS CIÊNCIAS DA SAÚDE. O evento marcou o lançamento da revista científica REVISE que contou com a presença de editores da Revista Baiana de Saúde Pública e da Revista Gueto. O evento aconteceu de 15 a 18 de novembro de 2017 no IFBA SAJ sob a direção da Universidade Federal do Recôncavo da Bahia.

Palavras-chave: Revista científica. Divulgação. Trabalhos científicos. Periódico.

\section{WORKSHOP OF TECHNOLOGICAL INNOVATION RESEARCH GROUPS IN HEALTH SCIENCES.}

\begin{abstract}
The paper records the conferences of the event international congress on technological innovation in health sciences: the sustainability of integrative practices in agroecology, more specifically the WORKSHOP conference of TECHNOLOGICAL INNOVATION RESEARCH GROUPS IN HEALTH SCIENCES. The event marked the launch of the scientific journal REVISE, which was attended by editors of Baiana Public Health periodic and Gueto periodic. The event took place from November 15 to 18, 2017 at IFBA SAJ under the direction of the Federal University of Recôncavo da Bahia.
\end{abstract}

Keywords: Scientific journal. Divulgation. Scientific work. Periodic.

Workshop de pesquisa. Revista Revise, vol 3, no fluxo contínuo, p.210-213. 

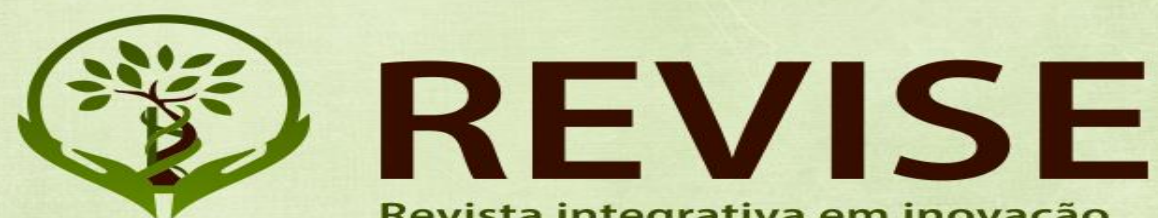

Revista integrativa em inovação tecnológica nas ciências da saúde

ISSN: 2179-6572
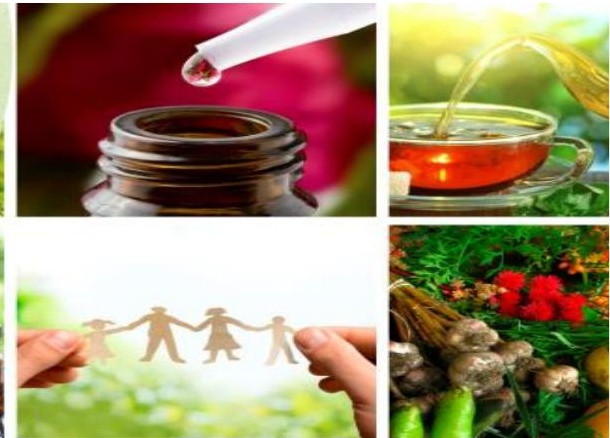

\section{INTRODUÇÃO}

No Workshop dos Grupos de pesquisa, o professor Júlio César dos Santos com sua estudante bolsista PIBEX Layane Assis Costa apresentaram a Revise - Revista Integrativa em Inovações Tecnológicas nas Ciências da Saúde, endereço eletrônico https://revistarevise.wordpress.com/ (a), como também realizaram um novo lançamento. Com o lançamento da revista, o congresso possibilitou de forma mais sustentável novas publicações em artigos científicos, resumos e resumos expandidos a respeito de temas conexos aos interesses dos pesquisadores/empreendedores em Agroecologia, Sustentabilidade e Medicina Tradicional.

O lançamento contou com a presença da representante da Revista Baiana de Saúde Pública e da Revista Gueto. No momento do lançamento a estudante Layane (PIBEX) recapitulou o primeiro número em 2008, em que seus editores se afastaram para o concurso de doutorado em outros estados. O lançamento é a retomada com a produção científica de alguns de seus integrantes anteriores.

O evento contemplou duas formas de divulgação e publicação dos trabalhos aprovados. A primeira delas foi a publicação na REVISE das conferências, minicursos como pré-print, os artigos para discussão, conforme endereço eletrônico https://revistarevise.wordpress.com/. Foi discutido a modalidade pré-print: publicar textos prévios, relatórios técnico-científicos para melhorar a teorização do artigo. Os pesquisadores presentes realizaram várias discussões da modalidade. Alguns pesquisadores informaram que revistas internacionais A1 ofertam a mesma modalidade. Outros pesquisadores estavam indecisos com a proposta do pré-print, pois dificilmente as pessoas reescrevem. No contra-argumento, pesquisadores explicaram que as revistas científicas, como a revista eletrônica da Faculdade de Ciências Econômicas da UFMG tem a seção de textos para discussão.

Workshop de pesquisa. Revista Revise, vol 3, no fluxo contínuo, p.210-213. 

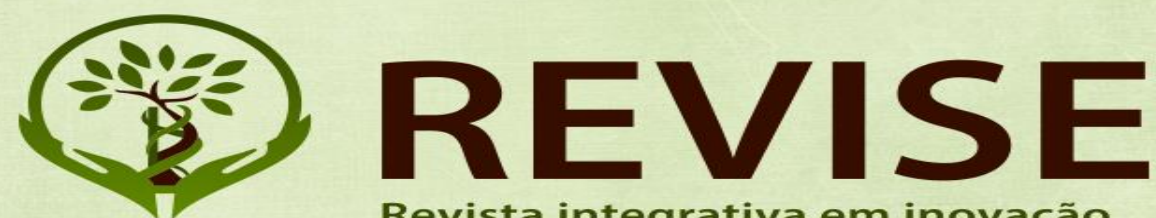

Revista integrativa em inovação tecnológica nas ciências da saúde

ISSN: 2179-6572
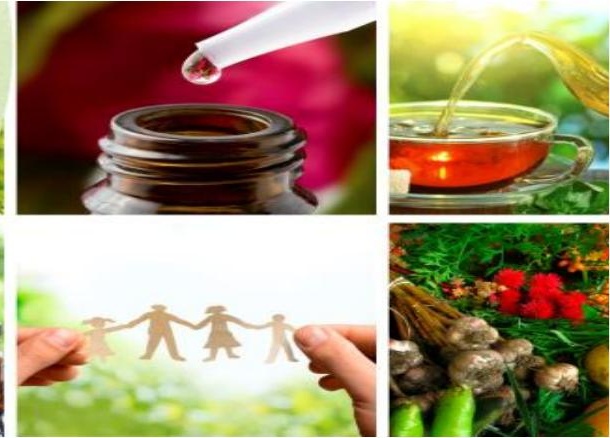

Os membros do comitê científico se tornaram avaliadores da REVISE ao final do Congresso, sendo que as melhores propostas de artigos completos tiveram seus artigos publicados conforme endereço eletrônico https://revistarevise.wordpress.com/sobre-2/. Com o lançamento da Revise durante o evento possibilitou fortalecer as redes de pesquisa em práticas integrativas, medicina integrativa, segurança alimentar com os membros dos diversos grupos de pesquisa nacionais e internacionais. Foram feitas articulações para que o comitê de avaliadores da REVISE tenha membros das comunidades tradicionais, pesquisadores e de instituições parceiras conforme endereço elrônico https://revistarevise.wordpress.com/sobre-2/. Entre outras discussões decidimos na oportunidade pela publicação dos anais do congresso na revista científica como primeiro número.

Também apresentou (b) a professora Joana Angélica Oliveira Molesini, Editora executiva da Revista Baiana de Saúde Pública. A professora mostrou o histórico da revista desde a sua fundação até os dias atuais; discutiu com outras revistas as possibilidades de ofertar espaços para textos para discussões, pré-print. A revista descartou o pré-print devido à necessidade do ineditismo para as publicações.

Tivemos apresentações de alguns grupos de pesquisa como do professor Júlio César dos Santos - Grupo de pesquisa SAED - Metodologias para a vida e Grupo de Pesquisa Pensamento e Cultura - UnB; Inês Macamo Raimundo do International Council for Sciences (ICSU); Regional Committee for Africa (RCA); Southern African Migration Program - SAMP e African Food Security Urban Network - AFSUN. Professora Sonia Maria Dalcomuni do Grupo de Pesquisa em Economia Criativa e Desenvolvimento da Indústria Joalheira - UFES; Economia da Inovação, Nanotecnologia, Energias Renováveis e Desenvolvimento Sustentável - UFES; Mayira Sojo-Milano, (mensagens virtuais - responsável pelo Prefácio na REVISE/Corpo Editorial) do projeto AMI RAVREDA; Carolina Gusmão Magalhães e Ionara Magalhaes de Souza do Grupo de

Workshop de pesquisa. Revista Revise, vol 3, no fluxo contínuo, p.210-213. 

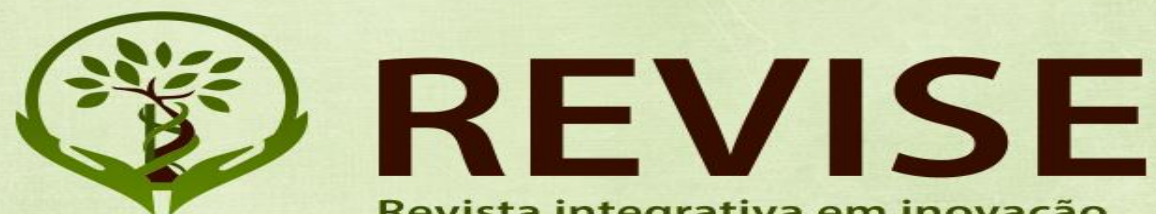

Revista integrativa em inovação tecnológica nas ciências da saúde

ISSN: $2179-6572$

Pesquisa Gueto - Grupo Unido para Educação e Trabalhos de Orientação - UFRB;

Marcílio Delan Baliza Fernandes, Ferlando Lima Santos, Carlos Alberto Soares da Costa - Grupo de Pesquisa de Bioprodutos e Processos Aplicados à Nutrição Humana BIONUTRI - UFRB; Grupo de pesquisa Propriedade Intelectual Transferência de Tecnologia e Inovação Tecnológica; Grupo de Pesquisa Doenças Infecciosas, Parasitárias e Crônicas - DIPAC - UFRB; Grupo de Pesquisa Alimentos e Saúde UFF; Estudos Avançados em Ciências e Tecnologias em Saúde - UFRB; Helene Paraskevi Anastasiou do Grupo de estudos sobre interdisciplinaridade GEINTER/UFRB; Grupo de Pesquisa SAED - Metodologias para a vida; Carlos Alberto Santos de Paulo - Grupo de Pesquisa NEGRAS - Núcleo de Estudos e Pesquisa em Gênero, Raça e Saúde - UFRB; Professora Doutora Ana Lucia Barreto da Fonseca dos grupos de pesquisa Meio Ambiente e Desenvolvimento Sustentável - UNIVASF; Comportamento, Desenvolvimento e Cultura - UFRB - participação de estudantes PIBIC; Maria Angélica Pereira de Carvalho Costa dos grupos de pesquisa Biotecnologia - UESB; Conservação e Diversificação no uso de Germoplasma de Fruteiras - EMBRAPA; Fruticultura Tropical - UFRB; INSECTA - UFRB; NBIO Núcleo de Melhoramento Genético e Biotecnologia - UFRB; Recursos Genéticos, Biotecnologia e Melhoramento da Mandioca - EMBRAPA. Os membros dos grupos realizaram vários diálogos para possibilitar a criação de um curso de especialização em Medicinas tradicionais com insumos agroecológicos.

Workshop de pesquisa. Revista Revise, vol 3, no fluxo contínuo, p.210-213. 